\title{
Status of the RFQ Beam Cooler for SPES project at LNL
}

\author{
Mario Maggiore ${ }^{1 \mathrm{a}}$, Anna M. Porcellato ${ }^{1}$, Sergey Stark $^{1}$, Francesca Chiurlotto ${ }^{1}$, Alessio Galatà ${ }^{1}$, \\ Antonio Dainelli ${ }^{1}$, Mauro De Lazzari ${ }^{1}$, Antonio Caruso ${ }^{2}$, Alberto Longhitano ${ }^{2}$ \\ ${ }^{1}$ INFN Laboratori Nazionali di Legnaro, I-35020 Legnaro, Italy \\ ${ }^{2}$ INFN Laboratori Nazionali del Sud, I-95123 Catania, Italy
}

\begin{abstract}
The SPES project is the new Radioactive Ion Beam facility under construction at the Laboratori Nazionali of Legnaro, Italy. In this framework, the realization of a new RFQ Beam Cooler (RFQBC) is in progress. This device allows to improve the quality of the low energy ion beams produced by the ISOL technique, granting a reduction of transverse emittance and energy spread. The RFQBC is an electromagnetic apparatus operating with oscillating electric fields needed to confine the very low energy $(\mathrm{eV})$ ions $(\mathrm{Q}=1+)$ which lose both the longitudinal and transversal momentum by making collisions with lighter atoms of the so called buffer gas $(\mathrm{He})$. This process provides the reduction of the transversal emittance up to 10 times of the initial value and it keeps the energy dispersion within 2-3 eV. The electromagnetic design of the RFQ section and the electrostatic layout of the injection and extraction regions have been done. The beam dynamics study is going on by means of dedicated codes which allow to take into account the interaction of the ions with the buffer gas needed to cool the beams. The RF group of LNS Catania is involved in the design of the RF system (500W) providing the oscillating high voltage (up to $3 \mathrm{kV}$ ) at frequency which varies in the range of 1-30 MHz. The preliminary design of the device started in 2011 in the framework of REGATA experiment of the $5^{\text {th }}$ scientific committee of INFN and it is carrying on within the COOLBEAM experiment.
\end{abstract}

\section{INTRODUCTION}

The SPES project [1] at Laboratori di Legnaro of INFN is aimed at the production of neutron-rich radioactive nuclei for nuclear physics experiment, by the Uranium fission at a rate of $10^{13}$ fission/s. The emphasis to neutron-rich isotopes is justified by the fact that this vast territory has been little explored. The facility is based on a high intensity proton cyclotron able to accelerate the beams up to $40 \mathrm{MeV}$ and a current of $300 \mu \mathrm{A}$. The impingement of the primary beam on the target, placed on a $40 \mathrm{kV}$ platform, provides radioactive ions with a current intensity up to $2 \mu \mathrm{A}$ in the mass range of 9160 AMU. A Wien filter and a couple of 90 deg magnets located downstream the source, make a first stage mass selection with $\mathrm{R} \sim 1 / 200$ and reduces the beam intensity to about $50 \mathrm{nA}$. The $\mathrm{Q}=1+$ ion beam is then delivered through the transport line with a geometric emittance of $30 \pi \cdot \mathrm{mm} \cdot \mathrm{mrad}$ at 40 $\mathrm{keV}$. The High Resolution Mass Spectrometer (HRMS) provides the separation of the radionuclides of interest from the isobaric contaminants up to a resolving power of 1/20000. In order to get this feature,

\footnotetext{
${ }^{a}$ Corresponding author: mario.maggiore@lnl.infn.it
} 
the HRMS is placed on a high voltage platform, and it needs of an input beam with low emittance (few $\pi \cdot \mathrm{mm} \cdot \mathrm{mrad}$ ) to improve the beam separation at slits position. A lower emittance has the further advantage of reducing the beam transport losses and, moreover, makes easier the detection of radioisotopes and increases the accuracy of the measurement of their properties. The devices usually adopted to reduce the emittance of low energy (few tens of $\mathrm{keV}$ ) ion beams are radio frequency quadrupoles cooler filled with some buffer gas [2].

\section{RFQ COOLER PHYSICS}

In an RFQ cooler the temperature of the ion beams is reduced via successive collisions with the atoms of a buffer gas. By applying the RF field to a quadrupole electrode structure, one can provide a radial force which counteracts the dispersion of the ions caused by the cooling process. The principle of the confinement is the same as in quadrupole mass filter. An RF voltage with amplitude $V$ and frequency $\omega_{\mathrm{RF}}$ is applied to the rods of a quadrupole in phase opposition. The motion of the ions in the RF quadrupole is governed by the so-called Mathieu parameter $q$ [3].

$$
q=\frac{4 \cdot Q \cdot V}{m \cdot r_{o} \cdot \omega_{R F}}
$$

Where $\mathrm{Q}$ is the charge and $\mathrm{m}$ the mass of the ion, and $\mathrm{r}_{\mathrm{o}}$ is one half of the distance between two opposite rods. The ion motion is stable with $0<\mathrm{q}<0.9$ [4]. The depth of the potential well is given by

$$
D=\frac{q \cdot V}{4}
$$

The so-called pseudopotential approximation is valid with low values of $q(<0.5)$ where, without interaction of the buffer gas, the ion motion can be regarded as simple harmonic oscillation at macromotion frequency

$$
\omega_{o}=\frac{q}{2 \cdot \sqrt{2}} \omega_{R F}
$$

The ions have additional oscillation, the so-called micromotion which comes from the driving RF field. The micromotion has the same frequency of the RF field and amplitude proportional to the electric field being zero in the axis and increasing linearly with the radius. At low values of $q$ the micromotion is just a ripple on the top of the macromotion, but above $q=0.5$ the micromotion amplitude becomes of the same order as the macromotion amplitude, and the two motions became indistinguishable. The best condition for an efficient ions thermalization is when $0<q<0.5$. An additional condition for an efficient cooling is that the buffer gas atoms are sufficiently lighter than the mass of the ions. It was demonstrated [4] that in the RFQ environment, the collisions of the ions with heavier mass result in a net ions heating whereas collisions with molecules lighter than the confined ions result in cooling towards the temperature of the background gas itself. In this case, the micromotion is only slightly modified in phase and amplitude while the main motion is dumped exponentially.

Once the ions are stopped and cooled within the buffer gas they must be dragged through the remaining gas in the path to the exit. This is accomplished by a longitudinal component of the electric field provided by DC potentials applied to the successive RFQ segments.

\section{STUDY AND DESIGN OF COOLER}

The RFQ beam cooler device is composed by 3 main sections: the deceleration system, which provides the reduction on the energy of the incoming beam from $40 \mathrm{keV}$ to some hundreds of $\mathrm{eV}$; the confinement and cooling section placed on a $39.8 \mathrm{kV}$ high voltage platform, where is located the RFQ and where the buffer gas is injected in order to get a pressure within $0.5 \div 3 \mathrm{~Pa}$; finally the acceleration 
section where the cooled beam is extracted and accelerated up to $40 \mathrm{keV}$. In this preliminary design phase the space charge effect on the transport of high intensity beams has been neglected.

\subsection{Injection and RFQ matching}

A system of four electrostatic lens decelerates the beam from $40 \mathrm{kV}$ to $200 \mathrm{eV}$ before the RFQ cooling section. The lens array gradually decelerates the ion beams to low energy, thus avoiding strong ionoptical effect that leads to harmful beam losses during the injection into the collisional ion guide. The conical shape of the two mid stage electrodes allows beam focusing and compensates the natural diverging effect due to the deceleration process. We chose such electrode configuration in order to achieve the $100 \%$ transmission efficiency between the injection and the RFQ sections. Figure 1 shows a sample plot of the trajectories of Cs 1+ ion through the electrode system.

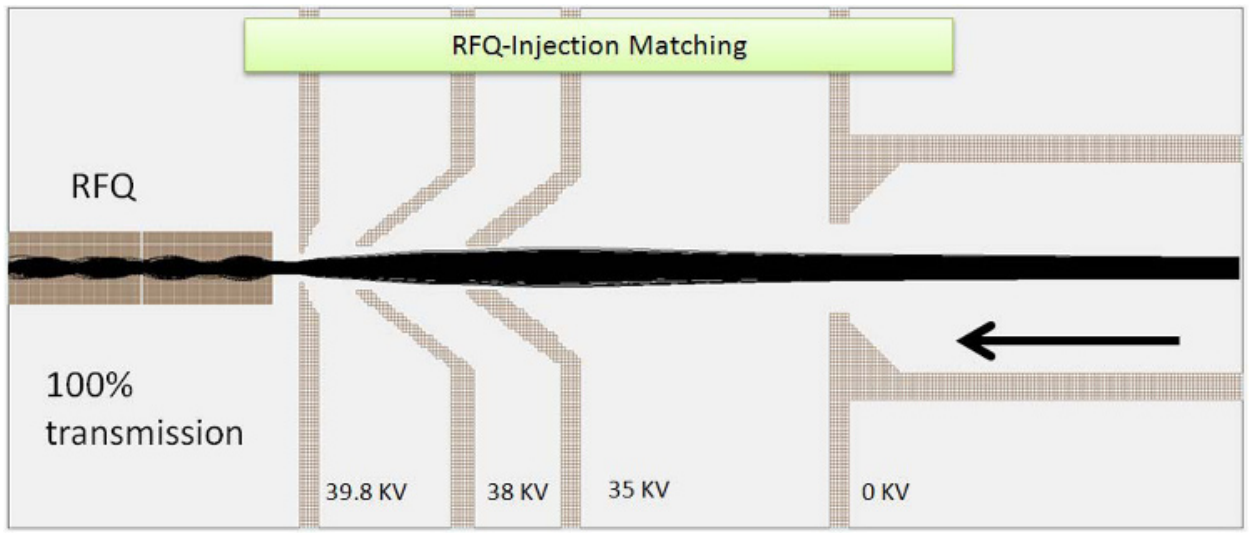

Figure 1. The SIMION beam transport simulation for the ${ }^{133} \mathrm{Cs}^{1+}$ beam along the deceleration system. This is composed by 2 stages of electrodes at different voltage. As shown the waist is placed as close as possible to the RFQ entrance in order to fully match the related acceptance.

\subsection{Confinement and Cooling Section}

The RF quadrupole consists of four cylindrical rods of $9 \mathrm{~mm}$ of diameter. The distance between the opposite pair of rods is $\Phi=8 \mathrm{~mm}$ The rods are $700 \mathrm{~mm}$ length and they are divided into 10 segments of $69.8 \mathrm{~mm}$ each. The segmentation allows to produce an axial field which provides the drag force needed to bring out the cooled beam. The total voltage applied along the segmentation is $100 \mathrm{~V}$.

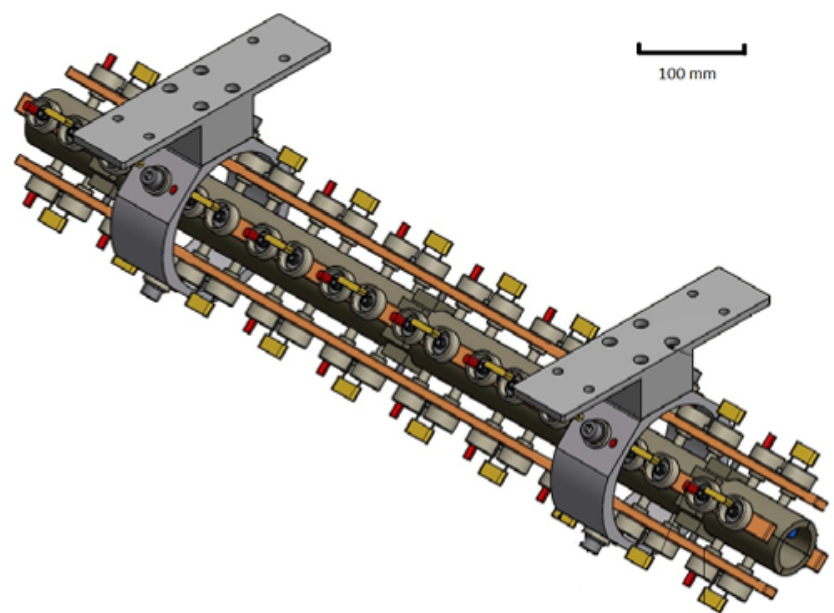

Figure 2. Layout of the quadrupole structure under construction at LNL. 
The applied RF voltage and the operating frequency depends on the ion mass delivered which varies within 9-160 AMU and on the current intensity of the incoming beam. For cooling a 50nA beam current, they range from $0.2 \div 1 \mathrm{kV}$ and $1 \div 30 \mathrm{MHz}$. The He at $293 \mathrm{~K}$ was chosen as buffer gas for ion cooling. The operational gas pressure varies from 0.5 to $3 \mathrm{~Pa}$, depending on the ion mass of the beam and on the RF voltage applied to the quadrupole. By the preliminary results given by the SIMION code using the hard sphere model to calculate the interaction gas-ion, we simulated the cooling process and we achieved a transverse emittance reduction of a factor 8-10 as shown in fig. 3 .

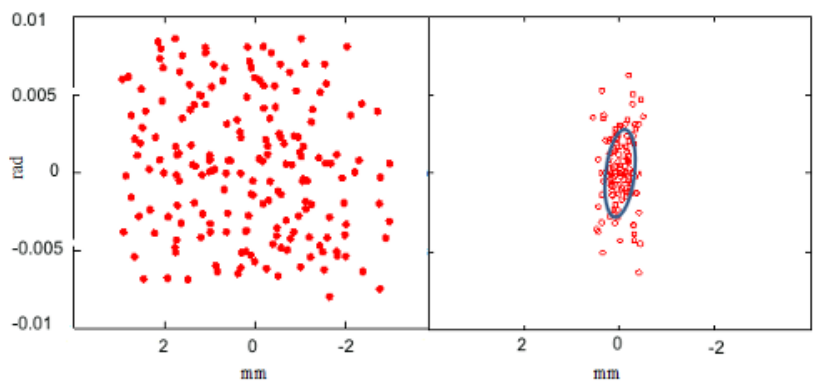

Figure 3. The plot shows the effect of the cooling process on the transversal emittance of ${ }^{133} \mathrm{Cs}^{1+}$ ion beam calculated by the SIMION code. On the left side the starting emittance of $30 \pi \mathrm{mm}$ mrad and on right side the reduced one by a factor 10 . The operational parameters are $V_{R F}=1.5 \mathrm{kV}, f_{R F}=5.57 \mathrm{MHz}, P_{H e}=2.5 \mathrm{~Pa}, q=0.22$, transmission $=90 \%$.

The differential pumping system is very crucial for obtaining optimal cooling and transmission efficiency. The gas leakage through the entrance apertures causes the energy degradation of the beam and the related beam losses due to the scattering with molecules of the residual gas. These harmful effect can be reduced by both the careful design of the differential pumping system and the optimization of the design of the injection and extraction electrodes and their placing with respect to the entrance and exit of the vacuum chamber where the RFQ is placed.

Following the cooling process through the RFQ, the ion beams exit through a $6 \mathrm{~mm}$ diameter aperture and then they recovered the initial energy by a two electrode stages.

\section{CONCLUSION}

The SPES project is being constructed at LNL and it foresees to start the first phase (driver and target commissioning) by the 2015. In the meantime the design of the beam transport line for the exotic species selection is almost completed, and all the devices (HRMS, charge breeder and related mass separator) are being designed. The RFQ beam cooler which is the key device to get the high resolution feature of the beam selection is under construction. The quadrupole and the main vacuum chamber are expected to be realised by the 2013 . In the 2014 the final assembly will be done at LNL.

\section{References}

1. A. Andrighetto et al. "The SPES project at LNL” AIP Conf. Proc. 1099 pp. 728-732.

2. R. B. Moore et al., Nucl. Instr. and Meth. B 204 (2003) 557.

3. E. Mathieu, Journal de Mathématique pure et appliquées, (1868),137-203

4. F.G. Major and H.G. Delmet, Phys. Rev. 170 (1968) 91. 